Aims. To examine the relationship between depression, anxiety and wellbeing in doctors.

Background. The relationship between doctor wellbeing and mental health diagnoses is not well evidenced in the literature. There is a lack of comparable measurement of wellbeing in doctors within the National Health Service, meaning the effectiveness of wellbeing interventions is unknown.

Method. A cross-sectional survey containing the PHQ9, GAD7 and WEMWBS questionnaires to measure depression, anxiety and wellbeing respectively, was advertised online nationally. The relationships between the total scores were explored using Spearman's rho correlation coefficients and Chi square tests. Thematic analysis of semi-structured interviews offered further insights.

Result. Sixty-seven doctors returned completed questionnaires. $29.9 \%$ had PHQ9 scores $>5$ and $41.8 \%$ had GAD7 scores $>5$. Therefore, over a quarter of the participants had a score that would suggest a management plan was needed for depression, and a third for anxiety. Moderate negative correlation between the total WEMWBS scores and the total PHQ, $\mathrm{rs}=-0.775, \mathrm{p}=0.00$, $\mathrm{N}=67$ and GAD7 scores $\mathrm{rs}=-0.724,, \mathrm{p}=0.00, \mathrm{~N}=67$ was seen. Statistically significant differences between those with low wellbeing scores (WEMWBS $<40$ ) and normal wellbeing scores (WEMWBS $\geq 40$ ) in relation to the need for a management plan for depression (PHQ9 > 10) X2 $(1, \mathrm{~N}=67)=12.395, \mathrm{p}=0.00$ and anxiety $(\mathrm{GAD} 7>10) \mathrm{X} 2(1, \mathrm{~N}=67)=5.611, \mathrm{p}=0.018$ were seen. The main themes identified from the interviews $(n=10)$ were the importance of social support outside of work, cynicism about an NHS plan check-in and a tendancy to neglect wellbeing until it has dipped.

Conclusion. There is a moderate negative correlation between anxiety, depression and wellbeing, but they are not opposites and separate measures for wellbeing should be used. It is clinically useful to note that only those with a WEMWBS score of $<45$ had a PHQ9 score suggesting the need for treatment of depression.

\section{Striving for better comunication - an audit}

\section{Eleanor Breen}

NHS Lanarkshire

doi: 10.1192/bjo.2021.86

Aims. The aim of this audit is to assess communication between the general and psychiatric hospital. This audit was prompted after a number of patients were transferred to Udston Hospital, a community hospital with two older adult acute mental health wards, with no written communication. This led to several significant issues including medication errors, ambiguity regarding patient escalation plans and uncertainty regarding what had been discussed with families.

Method. Over the course of one month eight patients were identified who had been transferred from the acute site to Udston Hospital. Three were new admissions to Udston, four were returning after treatment for physical illness, and one returned following assessment in ED. Data were collected by examining paper and electronic notes, and analysed using Excel. The results of this audit were discussed at the local clinical governance meeting. A 2nd cycle was performed. Eight transfers were identified. Four were returning after an assessment in ED, two were new admissions to Udston and two were returning after treatment for physical illness.

Result. Initial audit found that $38 \%$ of patients were transferred with their medical notes, $50 \%$ were transferred with no written documentation whatsoever, and none of the patients were transferred with a transfer letter. The second cycle found that $88 \%$ of patients had a transfer or discharge letter. $12 \%$ of patients came with no written documentation.

Conclusion. The initial audit found significant deficiencies in communication. Highlighting the need for all patients to have a transfer letter at a local management meeting seems to have led to an improvement. However, differences between the samples in the 1st and 2nd audit cycle could be distorting the results. Further audits would be useful given the small sample size and due to the differences between the sample populations.

\section{Neurodiversity in the teaching of the mental state examination: a pilot study of interactive mind-mapping seminars for the new ScotGem (Scottish graduate-entry medicine) students during the COVID-19 pandemic}

\author{
Kathleen Breslin ${ }^{1 \star}$, Sara Mohsen ${ }^{2}$ and Praveen Kumar ${ }^{2}$ \\ ${ }^{1}$ New Craigs Hospital, NHS Highland, University of Aberdeen and \\ ${ }^{2}$ New Craigs Hospital, NHS Highland \\ ${ }^{\star}$ Corresponding author.
}

doi: 10.1192/bjo.2021.87

Aims. Agility in educational delivery has been catalyzed in response to national restrictions mandated by the recent COVID-19 pandemic. Increased use of assistive technologies further aligns with the General Medical Council's aims that medical educators provide an 'accessible training experience'. The study examined medical students' receptiveness to different types of interactive teaching. Two undergraduate cohorts received teaching on the Mental State Examination, either socially-distanced delivered by traditional powerpoint or remotely by mind-mapping software on a tablet hand-held digital device. We required an effective program which would retain the popular interactive elements of Psychiatry teaching and promote inclusivity across students' diverse learning styles.

Method. Two cohorts of Year 2 students from the Universities of Dundee and St Andrew's Scottish Graduate-Entry Medicine (scotGEM) course took part in an Introduction to Psychiatry seminar which involved a presentation of the Mental State Examination. One was conducted in a face-to-face setting via traditional PowerPoint. The second was conducted via remoteconferencing with mindmaps of key concepts drawn and screenshared live to students as teaching progressed.

This was a qualitative study, with online links to questionnaires for 24 student participants across 5 domains. (1. The tutorial met my learning objectives, 2 . The format was suitable for me, 3 . The balance of theory and cases was suitable for me, 4 . The tutorial was of appropriate length, 5. I was satisfied with the performance) Response options included: strongly disagree, disagree, neutral, agree, strongly agree. A section was also included with openended questions pooled for thematic analysis.

Result. Response rate reached $>60 \%$ with $>80 \%$ respondents answering strongly agree across all domains. Thematic results demonstrated positive responses across both teaching sessions, with the interactive elements valued by students. Comments included: "great job was done with the delivery of the session considering it was online rather than in person"; "drawing element was fantastic"; "Good: interactivity of the session drawing and creativity element".

Conclusion. The Mental State Examination (MSE) via live-drawn mind-maps allows salient clinical information to be conceptualised in non-linear diagramatic format. This paediological approach can offer further access points across wide range of 